\title{
Simple Acupoints Prescription Flow Chart Based on Meridian Theory: A Retrospective Study in 102 Dogs
}

\author{
Jong-Ho Jeong, ${ }^{1}$ Joo-Young Song, ${ }^{2}$ Hyo-Gwon Jo, ${ }^{3}$ Ji-Min Kim, ${ }^{4}$ \\ Samuel-S. Yoon, ${ }^{5}$ Chul Park, ${ }^{6}$ Seunghyun Kim, ${ }^{7}$ Seong-Soo Roh, ${ }^{7}$ Bong Hyo Lee, ${ }^{7}$ \\ Chae Ha Yang, ${ }^{7}$ and Hee Young Kim ${ }^{8}$
}

\author{
${ }^{1}$ Boo-Young Animal Hospital, Gigeum-dong, Namyangju-si, Gyeonggi-do 472-080, Republic of Korea \\ ${ }^{2}$ Sarang Animal Hospital, Guro-3-dong, Gurogu, Seoul 152-053, Republic of Korea \\ ${ }^{3}$ Jakjeon 24 Hour Animal Hospital, Jakjeon 1-dong, Gyeyang-gu, Incheonsi, Gyeonggi-do 407-060, Republic of Korea \\ ${ }^{4}$ Jun Animal Hospital, Galsan-dong, Dongangu, Anyangsi, Gyeonggi-do 431-088, Republic of Korea \\ ${ }^{5}$ Twin City Animal Hospital, 869 South Street, Fitchburg, MA 01420, USA \\ ${ }^{6}$ Department of Veterinary Internal Medicine, College of Veterinary Medicine, Chonbuk National University, Jeonju-si, \\ Jeollabuk-do 561-756, Republic of Korea \\ ${ }^{7}$ College of Korean Medicine, Daegu Haany University, Daegu 706-828, Republic of Korea \\ ${ }^{8}$ Department of Physiology, College of Korean Medicine, Daegu Haany University, Daegu 706-828, Republic of Korea
}

Correspondence should be addressed to Hee Young Kim; hykim@dhu.ac.kr

Received 8 February 2013; Accepted 9 April 2013

Academic Editor: Younbyoung Chae

Copyright (C) 2013 Jong-Ho Jeong et al. This is an open access article distributed under the Creative Commons Attribution License, which permits unrestricted use, distribution, and reproduction in any medium, provided the original work is properly cited.

\begin{abstract}
To help the clinicians prescribe acupoints easily and effectively, we developed one simple flow chart to select acupoints. This study aimed to evaluate the usefulness of flow chart to select acupoints in dogs. Total 102 dogs showing intervertebral disc disease (IVDD) $(n=12)$, vomiting $(n=11)$, diarrhea $(n=2)$, abdominal pain $(n=5)$, cough $(n=66)$, or epilepsy $(n=6)$ received acupuncture treatment according to the chart, and its outcomes were evaluated as regards clinical symptoms, duration, treatment numbers, and recovery time. Dogs (8/8) with IVDD from grades I to III recovered over periods of 5 days to 6 weeks after 1-12 treatments, while 1/4 dogs with grade IV recovered over 7 weeks after 15 treatments. Vomiting dogs with acute/subacute $(n=8)$ and chronic symptoms $(n=3)$ required about 1 and 7 treatments to recover fully, respectively. All dogs $(n=5)$ with abdominal pain showed fast relief within 24 hours after acupuncture. Two diarrhea cases recovered over 2-9 days after 1-2 treatments. Fifty-four of 66 coughing dogs were recovered by 1-2 treatments. And 5 of 6 epilepsy dogs under a regular acupuncture treatment had no epileptic episode during followup of 12 months. These results suggest that this flow chart can help the clinicians prescribe acupoints effectively.
\end{abstract}

\section{Introduction}

Acupuncture had been used in human and veterinary practice for thousands years in Eastern Asia including China (called traditional Chinese medicine), Republic of Korea (called Korean medicine), Japan (called Kampo medicine), and other Asian countries until it was banned in their counties to promote Western medicine in the early 1900s. Since the visit of President Nixon to China in 1972 and articles about surgery in conscious patients under acupuncture anesthesia first hit the West, there has been an explosion of interest about acupuncture in the United States, Europe, and other countries. Veterinary acupuncture has also been resurrected and developed rapidly during the past 30 years. Veterinary acupuncture organizations have been established in most developed countries including those of North and South America, Europe, the Middle East, Australia and New Zealand, South Africa, Republic of Korea, and many Asian countries. Also, some veterinary schools have included class on acupuncture in their curriculum [1].

The number of certified veterinary acupuncturists and veterinarians wishing to study acupuncture has increased 
remarkably in the last decade. Now, many veterinarians and veterinary students spend much effort and expense to learn traditional oriental medical (OM) theory in an intensive course. They aim at integrating acupuncture into their practice but they discover quickly that OM theories are extremely complex and confusing to be applied in practice. Therefore, novices in the art science of veterinary acupuncture tend to rely strongly on "Cookbook Acupuncture," in which a routine set of acupoints is used to treat certain diseases. Though "Cookbook Acupuncture" is very useful for beginners, it is not specific for individual cases and its results are not as good as those of acupuncture adapted by experts for each specific case. Therefore, many practitioners using "Cookbook Acupuncture" become frustrated when they achieve outstanding results in some cases but no response in others.

To apply acupuncture most effectively, practitioners must make an OM diagnosis for each case, by using OM theory. That theory includes Yin-Yang, Zang-Fu organs, Channel (Meridian) Theory, and point indications. According to the OM diagnosis, two sets of acupoints are chosen: (a) main (essential) points and (b) helper (supporting) points. This combination of main and helper points is called an acupoints prescription or combination for each individual case. The correct choice of points plays a key role in acupuncture's success. When appropriated on combination, the main and helper acupoints produce synergic effects that boost the clinical efficacy of acupuncture. In contrast, some acupoints may counter the beneficial effects of the main acupoints through opposing actions [2-4]. For clinicians to select effective acupoints for individual cases efficiently, it is essential that they understand OM diagnosis, the properties and interactions of the Channels, the functions of each acupoint, and combination methods of their acupoints. However, it is difficult, especially for new acupuncturists, to comprehend OM theories completely, memorize all the information about acupoints, and build each treatment plan according to individual patient's condition. Therefore, we sought to develop for clinicians one simple acupuncture flow chart based on OM theories and diagnosis. Over a 5-year period, this flow chart has been modified through its clinical application. We now introduce one simple acupoints prescription chart and its clinical cases.

\section{Materials and Methods}

2.1. Acupoints Prescription Chart (Figure 1). The proposed chart was designed to select acupoints in a total of 5 steps.

In Step 1, according to the location of disease in the body (viz interior or exterior), two or three acupuncture points of 8 Extraordinary Channel points and 6 Command points were selected (Figures 2(a) and 2(b)).

In Step 2, one of 8 Influential points was chosen according to the body components (Zang, Fu, Muscle, Bone, Qi, Blood, Vessel, and Marrow) affected by disease (Figure 3).

In Step 3, the Back-Shu or Abdomen-Mu points sensitive to palpation were selected. To find the sensitive $S h u$ (Figure 4(a)) or $\mathrm{Mu}$ (Figure 4(b)) points on back and abdomen, the right hand palpated the skin on Back-Shu and Abdomen- $M u$ points while the left hand was positioned on abdomen. The points at which dogs exhibited the protective abdominal reflex, skin twisting, growling, grunting, and head-turning toward the palpated point during palpation were considered as sensitive Back-Shu or Abdomen$M u$ points, which were diagnosed as the affected internal organs [5] for the next Step 4. Those points were used as treatment points.

In Step 4, according to the diagnostic results from Step 3 or patients' main symptoms, the associated Channels were chosen (Figures 5(a) and 5(b)), and two main points, Yuan (source) point and $\mathrm{He}$ (sea) point, on the Channels were used for acupuncture. Some points on the Channel frequently were chosen according to the patient's condition, such as acute/ subacute ( $<7$ days), chronic ( $>7$ days), emergency, or joint pain.

Lastly, in Step 5, local points or empirical points for each case were added.

2.2. Cases $(n=102)$. The 102 dogs that received acupuncture treatment by 5 clinicians according to simple acupuncture flow chart were reviewed. Table 1 shows case signalment including age, sex, and disease duration. Ages of dogs varied from 3 months to 15 years (average age, 4.12 years). Forty-six percent (47/102) were males, and 54\% (55/102) were females. Purebred dogs accounted for 52\% (53/102) and were represented by 9 pure breeds (11 miniature Poodles, 14 Maltese, 6 Shih Tzus, 2 Chihuahuas, 1 Siberian husky, 8 Yorkshire terriers, 2 English cockers, 5 Pugs, and 4 miniature Schnauzers). The remaining 48\% (49/102) were mixed-breed dogs. Routine diagnostic tests including fecal examination, urinalysis, blood tests, and/or radiography were performed in private practice to determine the possible cause.

Acupuncture was prohibited in cases with evidence of foreign body on radiography or infectious viral disease (distemper or parvovirus) by commercial ELISA kits. All except epilepsy cases received acupuncture treatment only without conventional Western medicine. Cases were classified into 6 groups, according to main symptoms: intervertebral disc disease (IVDD) $(n=12)$, vomiting $(n=11)$, diarrhea $(n=2)$, abdominal pain $(n=5)$, cough $(n=66)$, and epilepsy $(n=6)$ (Table 1$)$. IVDD was further classified as grade I to IV: grade I = no neurologic signs except back pain, grade II = conscious proprioceptive deficit and ambulatory paraparesis, grade III = nonambulatory paraparesis, and grade IV = nonambulatory paraparesis with loss of deep pain perception [6]. Gastrointestinal disorders including vomiting, diarrhea, and abdominal pain were classified by the duration to clinical presentation as acute ( $<2$ days), subacute (2-7 days), or chronic ( $>7$ days). Application of acupuncture for cough was restricted to subacute cases (duration, 2-7 days), since acute cough was often a self-limiting problem that may resolve without any symptomatic/supportive therapy, or chronic cases had a risk of severe bacterial infection without antibiotics, subsequently death. In idiopathic epilepsy, one dog was 3 months at onset of epileptic seizure and the remaining 5 dogs were over 1 year, at a frequency of $2-4 /$ month under control of phenobarbital and potassium bromide therapy. Five of the 6 dogs showed generalized and symmetrical seizure and 
STEP 1: Find the location of disease [combined therapy of 8 extraordinary channel points and 6 command points]

\begin{tabular}{|c|l|c|l|}
\hline \multirow{2}{*}{ Exterior } & Location of disease & Points & \multicolumn{1}{c|}{ Add command points } \\
\cline { 2 - 3 } & Lateral aspects & SI3-BL62 & \multirow{2}{*}{ ·add ST36 for gastrointestinal disorders, } \\
\cline { 2 - 3 } Interior & Heart and gastrointestinal & TH5-GB41 & PC6 for urogenital disorders \\
\cline { 2 - 3 } & Respiratory and urological & PL40 for back disorders \\
& add LI4 for face, eye, nasal disorders \\
\hline
\end{tabular}

STEP 2: Find the affected area [8 influential points therapy]

\begin{tabular}{|l|l|l|}
\hline \multicolumn{1}{|c|}{ The affected area } & Point & \multicolumn{1}{c|}{ OM name } \\
\hline Liver, heart (pericardium), spleen (pancreas), lung, kidney & LV13 & Zang (Yin organs) \\
\hline Stomach, small intestine, large intestine, bladder & CV12 & Fu (Yang organs) \\
\hline Muscle-related diseases & GB34 & Ji (muscle) \\
\hline Bone-related diseases, especially in forelimb & BL11 & Gu (bone) \\
\hline Thoracic disease (mainly, respiratory) & CV17 & Qi (life/vital energy) \\
\hline Blood-related disorders & BL17 & Xue (blood) \\
\hline Vascular or circulatory disorders & LU9 & Mai (vessels) \\
\hline Neurological diseases, bone marrow disorders, fracture & GB39 & Sui (marrow) \\
\hline
\end{tabular}

STEP 3: Find sensitive back-Shu or abdomen- $M u$ points when palpated [Shu-Mu points therapy]

\begin{tabular}{|c|c|c|c|c|c|c|c|c|c|c|c|c|}
\hline Point & $\begin{array}{c}\text { Lung } \\
(\mathrm{LU})\end{array}$ & $\begin{array}{c}\text { Heart } \\
(\mathrm{HT})\end{array}$ & $\begin{array}{c}\text { Pericardium } \\
(\mathrm{PC})\end{array}$ & $\begin{array}{c}\text { Spleen } \\
(\mathrm{SP})\end{array}$ & $\begin{array}{c}\text { Kidney } \\
(\mathrm{KI})\end{array}$ & $\begin{array}{c}\text { Liver } \\
(\mathrm{LV})\end{array}$ & $\begin{array}{c}\text { Large } \\
\text { intestine } \\
(\mathrm{LI})\end{array}$ & $\begin{array}{c}\text { Small } \\
\text { intestine } \\
(\mathrm{SI})\end{array}$ & $\begin{array}{c}\text { Triple } \\
\text { heater }\end{array}$ & $\begin{array}{c}\text { Stomach } \\
(\mathrm{ST})\end{array}$ & $\begin{array}{c}\text { Bladder } \\
(\mathrm{BL})\end{array}$ & $\begin{array}{c}\text { Gall } \\
\text { bladder }(\mathrm{GB})\end{array}$ \\
\hline Back-Shu point & BL13 & BL15 & BL14 & BL20 & BL23 & BL18 & BL25 & BL27 & BL22 & BL21 & BL28 & BL19 \\
\hline Abdomen- $M u$ point & LU1 & CV14 & CV17 & LV13 & GB25 & LV14 & ST25 & CV4 & CV5 & CV12 & CV3 & GB24 \\
\hline
\end{tabular}

Step 4: choose the channel and main points on the channel according to main symptoms [channel points therapy]

\begin{tabular}{|c|c|c|c|c|c|c|c|c|c|c|c|c|c|}
\hline \multicolumn{2}{|c|}{$\begin{array}{c}\begin{array}{c}\text { Location of } \\
\text { symptoms }\end{array} \\
\end{array}$} & \multicolumn{6}{|c|}{ Interior } & & \multicolumn{5}{|c|}{ Exterior } \\
\hline \multirow{3}{*}{\multicolumn{2}{|c|}{ Specific area }} & Pancreas & \multirow{3}{*}{ 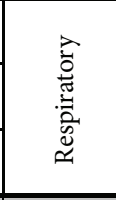 } & \multirow{3}{*}{\begin{tabular}{|c|} 
Liver \\
$\begin{array}{c}\text { Muscle, } \\
\text { tendon }\end{array}$ \\
$\begin{array}{l}\text { Urogenital } \\
\text { (exterior) }\end{array}$ \\
\end{tabular}} & \multirow{3}{*}{ 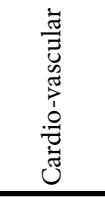 } & \multirow{3}{*}{ 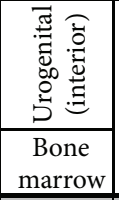 } & \multirow{3}{*}{ 氶 } & \multicolumn{2}{|c|}{ Front aspects } & \multicolumn{2}{|c|}{ Lateral aspects } & \multicolumn{2}{|c|}{ Posterior aspects } \\
\hline & & \begin{tabular}{|c|} 
Gastro- \\
intestinal \\
\end{tabular} & & & & & & \multirow{2}{*}{$\begin{array}{c}\text { Gastro- } \\
\text { intestinal }\end{array}$} & Face & \multirow{2}{*}{ Ear } & \multirow{2}{*}{ Ear } & \multirow{2}{*}{$\begin{array}{c}\text { Thoraco- } \\
\text { lumbar } \\
\text { spine }\end{array}$} & Scapula \\
\hline & & Blood & & & & & & & Tooth & & & & Neck \\
\hline \multirow{2}{*}{$\begin{array}{c}\text { Point } \\
\text { indication }\end{array}$} & $\begin{array}{c}\text { Channel } \\
\text { OM name }\end{array}$ & $\begin{array}{c}\text { Hindlimb } \\
\text { TaiYin }\end{array}$ & \begin{tabular}{|c} 
Forelimb \\
TaiYin
\end{tabular} & $\begin{array}{c}\text { Hindlimb } \\
\text { JueYin }\end{array}$ & \begin{tabular}{|c|} 
Forelimb \\
JueYin
\end{tabular} & \begin{tabular}{|l|}
$\begin{array}{l}\text { Hindlimb } \\
\text { ShaoYin }\end{array}$ \\
\end{tabular} & \begin{tabular}{|l|} 
Forelimb \\
ShaoYin \\
\end{tabular} & \begin{tabular}{|l|} 
Hindlimb \\
YangMing
\end{tabular} & $\begin{array}{c}\text { Forelimb } \\
\text { Yangming }\end{array}$ & $\begin{array}{l}\text { Hindlimb } \\
\text { ShaoYang }\end{array}$ & $\begin{array}{l}\text { Forelimb } \\
\text { ShaoYang }\end{array}$ & $\begin{array}{l}\text { Hindlimb } \\
\text { TaiYang }\end{array}$ & $\begin{array}{l}\text { Forelimb } \\
\text { Tai Yang }\end{array}$ \\
\hline & Organ & SP & LU & LV & PC & KI & HT & ST & LI & GB & $\mathrm{TH}$ & BL & SI \\
\hline \multirow{2}{*}{$\begin{array}{c}\text { Main } \\
\text { (essential) }\end{array}$} & Yuan (source) & SP3 & LU9 & LV3 & PC7 & $\mathrm{KI} 3$ & HT7 & ST42 & LI4 & GB40 & TH4 & BL64 & SI4 \\
\hline & $\mathrm{He}$ (sea) & SP9 & LU5 & LV8 & PC3 & KI10 & HT3 & ST36 & LI11 & GB34 & TH10 & BL40 & SI8 \\
\hline Acute & $X i$ (cleft) & SP8 & LU6 & LV6 & PC4 & KI5 & HT6 & ST34 & LI7 & GB36 & TH7 & BL63 & SI6 \\
\hline Chronic & $\begin{array}{c}\text { Luo } \\
\text { (connection) }\end{array}$ & ST40 & LI6 & GB37 & TH5 & BL58 & SI7 & SP4 & LU7 & LR5 & PC6 & KI4 & HT5 \\
\hline Emergency & Jing (well) & SP1 & LU11 & LV1 & PC9 & KI1 & HT9 & ST45 & LI1 & GB44 & TH1 & BL67 & SI1 \\
\hline Heat signs & Ying (spring) & SP2 & LU10 & LV2 & PC8 & KI2 & HT8 & ST44 & LI2 & GB43 & TH2 & BL66 & SI2 \\
\hline Joint pain & Shu (stream) & SP3 & LU9 & LV3 & PC7 & $\mathrm{KI} 3$ & HT7 & ST43 & LI3 & GB41 & TH3 & BL65 & SI3 \\
\hline Cold signs & Jing (river) & SP5 & LU8 & LV4 & PC5 & KI7 & HT4 & ST41 & LI5 & GB38 & TH6 & BL60 & SI5 \\
\hline
\end{tabular}

STEP 5: Empirical, local or Ashi points

\begin{tabular}{|c|c|c|c|c|c|c|c|c|c|c|}
\hline Emergency & $\begin{array}{l}\text { Tonification } \\
\text { effect }\end{array}$ & Fever & Head & Neck & Eye & $\begin{array}{l}\text { Lower } \\
\text { jaw }\end{array}$ & Cough & $\begin{array}{c}\text { Humeral } \\
\text { joint }\end{array}$ & $\begin{array}{l}\text { Lumbar } \\
\text { \& sacral }\end{array}$ & Diarrhea \\
\hline $\begin{array}{c}\text { GV26, KI1, HT9, } \\
\text { PC9 }\end{array}$ & $\begin{array}{l}\text { LI4, LI11, } \\
\text { LV3, ST36 }\end{array}$ & $\begin{array}{l}\text { ear tip, tail tip, } \\
\text { GV14 }\end{array}$ & $\begin{array}{l}\text { GV20, GB20, } \\
\text { GV16, Yintang }\end{array}$ & $\begin{array}{c}\text { GB20, BL10 } \\
\text { GV16 }\end{array}$ & $\begin{array}{l}\text { ST1, L1, } \\
\text { GB1 }\end{array}$ & ST7 & CV22 & $\begin{array}{l}\text { LI15, } \\
\text { TH14 }\end{array}$ & Baihui & GV1 \\
\hline
\end{tabular}

Figure 1: Acupoints prescription flow chart. 


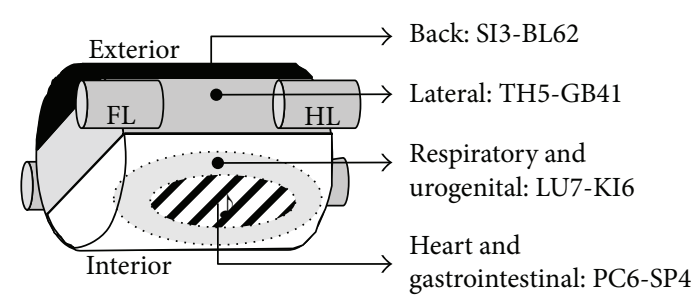

(a)

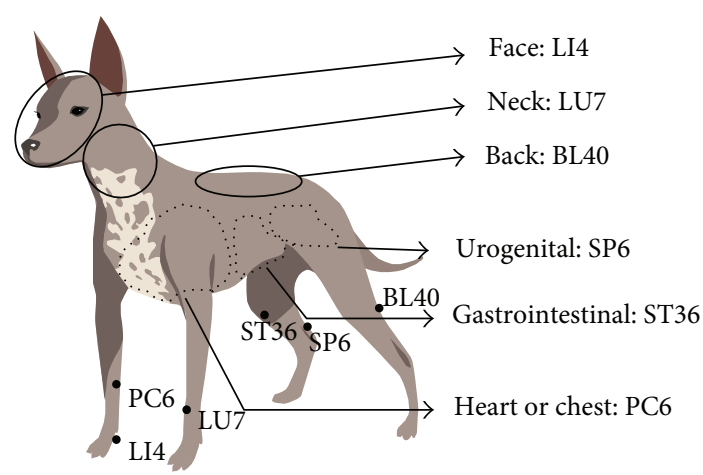

(b)

FIgURE 2: 8 Extraordinary Channel points and 6 Command points (Step 1). (a) Clinical indications of 8 Extraordinary Channel points. The body was simply divided into 4 areas of exterior-back aspects, exterior-lateral aspects, interior-heart/gastrointestinal and interiorrespiratory/urogenital systems, and paired acupoints (underlined) were used for disorders of their corresponding area. (b) Indication of 6 Command points. FL, forelimb; HL, hind limb.

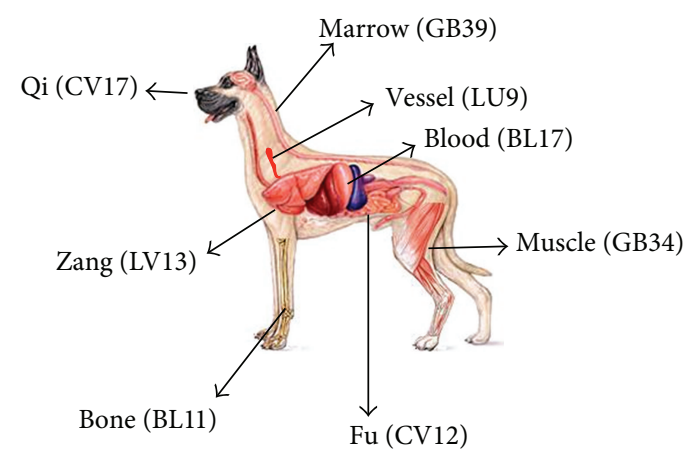

Figure 3: 8 Influential points (Step 2). Based on OM, body consists of 8 components and each of them can be controlled by its key point (underlined).

one was seen to have unilateral focal motor activity of the head which spread to unilateral limbs.

2.3. Acupuncture Treatment. Acupuncture was performed by 5 veterinarians in their own practice. After selecting acupoints according to the flow chart (Figure 1), acupuncture needles (stainless steel, $0.24-0.30 \mathrm{~mm}$ in diameter, $15-40 \mathrm{~mm}$ in length) were inserted, as described in text [5] and left for 15-20 minutes with/without manipulation. Acupuncture was applied 2-3 times/week. Clinical followup was determined either by a phone call to the owners at the time of this study or by return of the dog to the veterinary hospital. Treatment was discontinued if the owner stated the symptoms had ceased and if main symptoms were apparently disappeared on laboratory or physical examination.

\section{Results}

3.1. IVDD $(n=12)$. The age of the dogs varied from 2 to 9 years with an average age of 3.6 years. The affected breeds were miniature Poodle $(n=3)$, Yorkshire terrier $(n=2)$, miniature Schnauzer $(n=2)$, Shih Tzu $(n=1)$, and mixedbreed $(n=4)$. Spinal palpation pain (hyperesthesia), when assessed by manual compression along the thoracolumbar spine, was detected at one or more levels of T11-T12 $(n=2)$, T12-T13 $(n=6)$, T13-L1 $(n=5)$, L1-L2 $(n=4)$, L2-L3 $(n=1)$, and L3-L4 $(n=1)$. In cases with severe symptoms (grade III or IV), diffuse back pain over two spinal levels was frequently observed. On plain radiographic examination, narrowed intervertebral disk space $(n=3)$, osteophyte formation $(n=1)$, or mineralized intervertebral disk $(n=2)$ was found in grade III or IV cases. According to the flow chart (Figure 1), the following points were chosen for acupuncture: SI3, BL62, and BL40 (in Step 1), GB34 (in Step 2), Back-Shu points at levels showing hyperesthesia on the spinal palpation (in Step 3), and BL40 and BL60 (in Step 4). Acupuncture was performed 2-3 times a week. Cases that returned to normal or cases with non-ambulatory paraparesis (grade III or IV) that improved to be able to walk and void urine and feces without assistance were considered to be recovered. All dogs $(8 / 8,100 \%)$ from grades I to III recovered over periods of 5 days to 6 weeks after 1-12 treatments, while only 1/4 dogs (25\%), diagnosed as grade IV, recovered over 7 weeks after 15 treatments.

3.2. Vomiting $(n=11)$. The vomiting cases, diagnosed as unknown causes from diagnostic tests or not responsive to initial managements of food withdrawals and antiemetics for 1-3 days, were subjected to this acupuncture treatment. They were young ( $n=1,6$ months) or young adult dogs $(n=$ 10 , average age $=3.2 \mathrm{yrs}$ ) and showed commonly vomiting and inappetance. The acupoints were chosen based on the flow chart: PC6, SP4, and ST36 (in Step 1), LV13 and CV12 (in Step 2), sensitive Back-Shu, and Abdomen-Mu points to palpations (in Step 3), ST36, ST42, SP3, and SP9 (in Step 4). Acute vomiting cases ( $<2$ days; $n=6$ ) were well responsive to this acupuncture treatment. Interestingly, 3 of them began to eat food within $1 \mathrm{hr}$ after withdrawals of acupuncture needles and did not show any vomiting. In subacute cases (27 days; $n=2$ ) that showed vomiting 3-5 times/day and were 


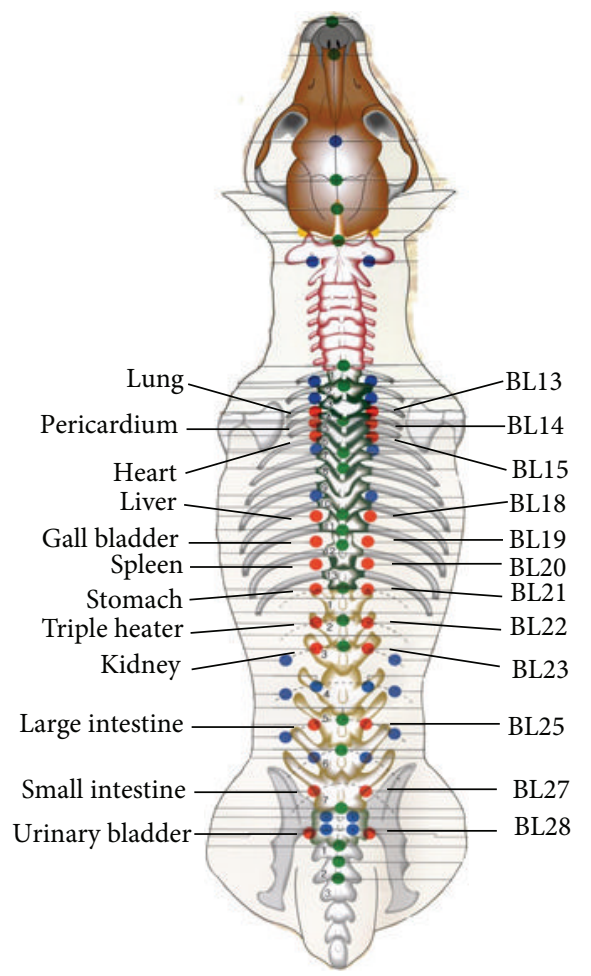

(a)

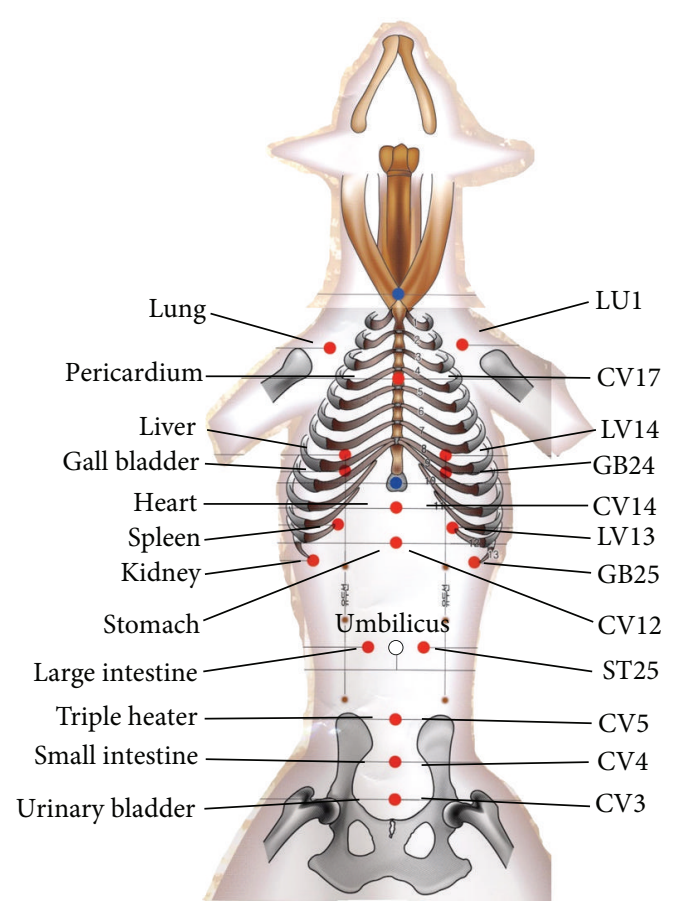

(b)

Figure 4: Back-Shu and Abdomen-Mu points (Step 3). (a) Back-Shu points (red color points) and internal organs. (b) Abdomen-Mu points (red color points) and internal organs.

TABLE 1: Case signalment.

\begin{tabular}{|c|c|c|c|c|c|c|}
\hline \multirow{2}{*}{ Diseases } & \multirow{2}{*}{ Severity } & \multirow{2}{*}{ Duration } & \multirow{2}{*}{ Case $(n)$} & \multicolumn{2}{|c|}{ Sex } & \multirow{2}{*}{ Age (year) } \\
\hline & & & & Male & Female & \\
\hline \multirow{4}{*}{ IVDD } & Grade I & & 1 & 1 & & 3.00 \\
\hline & Grade II & & 1 & 1 & & 2.00 \\
\hline & Grade III & & 6 & 2 & 4 & 2.87 \\
\hline & Grade IV & & 4 & 2 & 2 & 5.25 \\
\hline \multirow{3}{*}{ Vomiting } & Acute & $<2$ days & 6 & 2 & 4 & 2.77 \\
\hline & Subacute & 2-7 days & 2 & & 2 & 3.50 \\
\hline & Chronic & $>7$ days & 3 & 3 & & 3.00 \\
\hline \multirow{3}{*}{ Diarrhea } & Acute & $<2$ days & 1 & & 1 & 1.50 \\
\hline & Subacute & 2-7 days & 1 & 1 & & 0.60 \\
\hline & Chronic & $>7$ days & & & & \\
\hline \multirow{3}{*}{ Abdominal pain } & Acute & $<2$ days & 5 & 2 & 3 & 2.54 \\
\hline & Subacute & 2-7 days & & & & \\
\hline & Chronic & $>7$ days & & & & \\
\hline \multirow{3}{*}{ Cough } & Acute & $<2$ days & & & & \\
\hline & Subacute & 2-7 days & 66 & 30 & 36 & 4.55 \\
\hline & Chronic & $>7$ days & & & & \\
\hline \multirow{2}{*}{ Epilepsy } & & $<1$ year & 1 & 1 & & 0.6 \\
\hline & & $>1$ year & 5 & 2 & 3 & 5.76 \\
\hline Total & & & 102 & 47 & 55 & 3.75 \\
\hline
\end{tabular}




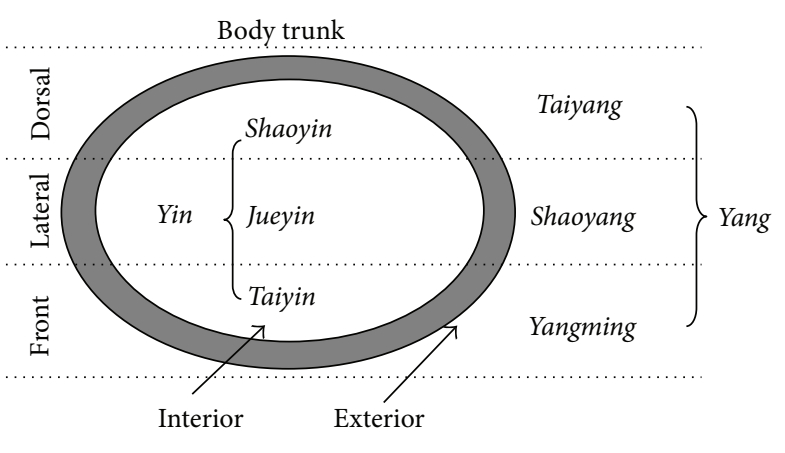

(a)

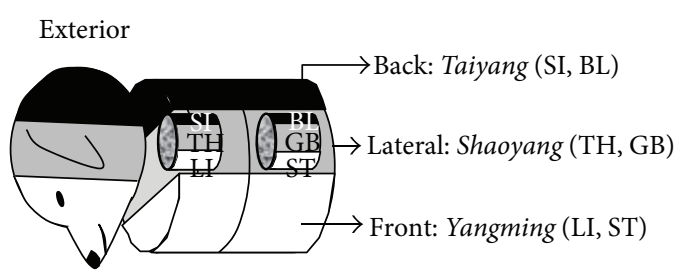

(b)

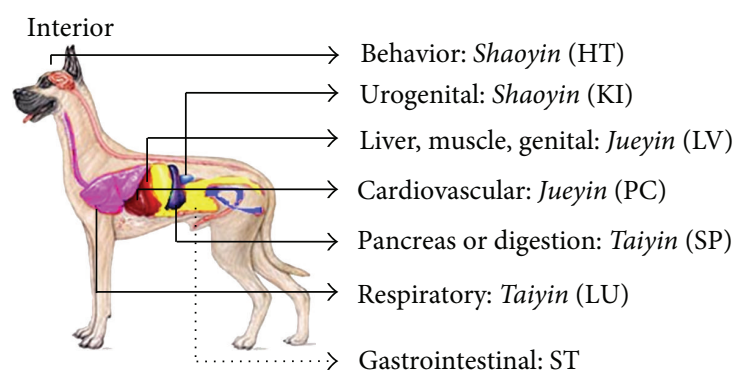

(c)

Figure 5: Channels and their clinical indications (Step 4). (a) Ancient anatomical terms "3 Yangs-3 Yins" and body. Yang and Yin represent exterior and interior of body, respectively. The Yang (exterior of body) is divided into 3 sub-Yangs, namely, Yangming (front), Shaoyang (lateral), and Taiyang (dorsal or back). On the other hand, the Yin (interior of body) is divided into 3 sub-Yins, namely, Taiyin (front organs; lung, spleen), Jueyin (middle organs; pericardium, liver), and Shaoyin (dorsal organs; heart, kidney). (b) Three Yang Channels and Exterior. External body is divided front, lateral and back, and their associated Channels (underlined) were used for disorders of each area. (c) Three Yin Channels and Interior. Each Yin Channel is used for treatment of their specific internal organs (a and c). Of the Yang Channels, the stomach (ST) Channel is especially useful to treat gastrointestinal disorders. The words in Italic are Chinese Pinyin. LI, large intestine Channel; ST, stomach Channel; TH, triple heater Channel; GB, gall bladder Channel; SI, small intestine Channel; BL, urinary bladder Channel; LU, lung Channel; SP, spleen Channel; PC, pericardium Channel; LV, liver Channel; HT, heart Channel; KI, kidney Channel.

not responsive to initial treatment of food withdrawals (first $24 \mathrm{hr}$ ) and metoclopramide, one or two vomitings were noted up to $12 \mathrm{hr}$ after first acupuncture and thereafter no more vomiting episodes. Chronic cases ( $>7$ days; $n=3$ ) suffered from sporadic vomiting of 2-3 times/day, frequently after food intake, recovered over 18.67 days after 6.7 times treatments and required longer periods and more treatments than acute and subacute cases.

3.3. Diarrhea $(n=2)$. A 1.5 -month-old female Poodle dog (acute case) was presented with acute watery diarrhea of 2 episodes within $12 \mathrm{hr}$. Since the patient still kept normal appetite without dehydration and pyrexia and owner was most favorable toward alternative medicine use, acupuncture was first tried without routine laboratory examinations or Western medicine. The following acupoints were used: PC6, SP4, and ST36 (in Step 1), LV13, and CV12 (in Step 2), sensitive Back-Shu and Abdomen-Mu points to palpations (in Step 3), ST42, ST36, SP3, and SP9 (in Step 4), and GV1 (in the last Step 5). Increase of stools consistency and decrease of stools frequencies were observed within $48 \mathrm{hr}$ after first acupuncture treatment. She returned to normal over 9 days after two acupuncture treatment. A 6-month-old male mixed dog (subacute case) was presented with acute onset $(<2$ days) of mild diarrhea, poor appetite, and frequent vomiting. When presented, a complete blood count and routine serum chemistry levels were within normal limits and fecal examinations including parvovirus, distemper virus $w$ and parasites were also normal. The patient was first treated with loperamide and amoxicillin for 5 days, but the diarrhea persisted. Acupuncture was then applied at the same points as those of acute diarrhea case. He began to recover appetite on the day of acupuncture treatment and returned to normal consistent feces 2 days after acupuncture treatment.

3.4. Abdominal Pain $(n=5)$. The cases ( 2 Maltese, 1 Pug, 1 Cocker spaniel, and 1 miniature Poodle) were initially presented with acute abdominal discomfort, increasing abdominal pain, inappetance, and/or bloating, and they all were lesser than 2 days at duration. Physical examination was normal, except abdominal pain. There was no radiographic evidence for foreign bodies. Acupuncture was performed at the acupoints selected according to the flow chart. They all returned to normal within 24 hours after one acupuncture treatment. Interestingly, when food was offered $30 \mathrm{~min}$ after withdrawal of acupuncture needles, $4 / 5$ cases began to eat immediately and did not show any abdominal pain on palpation. 
TABLE 2: The acupoints selected according to flow chart and treatment outcome.

\begin{tabular}{|c|c|c|c|c|c|c|}
\hline Diseases & Severity & $\begin{array}{l}\text { Case } \\
(n)\end{array}$ & The used acupoints & $\begin{array}{c}\text { Treatment } \\
\text { numbers }\end{array}$ & $\begin{array}{l}\text { Recovery } \\
\text { times (day) }\end{array}$ & $\begin{array}{c}\text { Recovered } \\
\text { animals/cases }\end{array}$ \\
\hline \multirow{4}{*}{ IVDD } & Grade I & 1 & \multirow{4}{*}{$\begin{array}{l}\text { Step 1: SI3, BL62, BL40 } \\
\text { Step 2: GB34 } \\
\text { Step 3: sensitive Back-Shu points } \\
\text { Step 4: BL40, BL64 (BL Channel) } \\
\text { Step 5: Baihui, Ashi points near lesion along BL Channel }\end{array}$} & 1.0 & 5.00 & $1 / 1$ \\
\hline & Grade II & 1 & & 8.0 & 49.00 & $1 / 1$ \\
\hline & Grade III & 6 & & 12.5 & 38.67 & $6 / 6$ \\
\hline & Grade IV & 4 & & 15.0 & 49.00 & $1 / 4$ \\
\hline Vomiting & $\begin{array}{l}\text { Acute } \\
\text { Subacute } \\
\text { Chronic }\end{array}$ & $\begin{array}{l}6 \\
2 \\
3\end{array}$ & $\begin{array}{l}\text { Step 1: PC6, SP4, ST36 } \\
\text { Step 2: LV13, CV12 } \\
\text { Step 3: sensitive Back-Shu and/or Abdomen-Mu points } \\
\text { Step 4: ST36, ST42, SP3, SP9 (ST, SP Channels) }\end{array}$ & $\begin{array}{l}1.0 \\
1.0 \\
6.7\end{array}$ & $\begin{array}{c}0.84 \\
1.00 \\
18.67\end{array}$ & $\begin{array}{l}6 / 6 \\
2 / 2 \\
3 / 3\end{array}$ \\
\hline \multirow{3}{*}{ Diarrhea } & Acute & 1 & \multirow{3}{*}{ (Same as those of vomiting) $+\mathrm{GV} 1$} & 2.0 & 9.00 & $1 / 1$ \\
\hline & Subacute & 1 & & 1.0 & 2.00 & $1 / 1$ \\
\hline & Chronic & 0 & & & & \\
\hline Abdominal pain & Acute & 5 & (Same as those of vomiting) & 1.0 & 0.62 & $5 / 5$ \\
\hline Cough & $\begin{array}{l}\text { Acute } \\
\text { Subacute } \\
\text { Chronic }\end{array}$ & $\begin{array}{c}0 \\
66 \\
0\end{array}$ & $\begin{array}{l}\text { Step 1: LU7, KI6, LI4 } \\
\text { Step 2: CV17 or LV13 } \\
\text { Step 3: sensitive Shu and/or } M u \text { points } \\
\text { Step 4: LU5, LU9 (LU Channel) } \\
\text { Step 5: CV22 }\end{array}$ & 1.9 & 7.02 & $54 / 66$ \\
\hline Epilepsy & $\begin{array}{l}<1 \text { year } \\
>1 \text { year }\end{array}$ & $\begin{array}{l}1 \\
5\end{array}$ & $\begin{array}{l}\text { Step 1: SI3, BL62 for posterior brain lesion or TH5, GB41 for } \\
\text { unilateral brain lesion } \\
\text { Step 2: GB39 } \\
\text { Step 3: sensitive Shu and/or Mu points } \\
\text { Step 4: SI4, SI8, BL40, BL64 for posterior brain lesion (SI, BL } \\
\text { Channels) or TH4, TH10, GB34, GB40, for unilateral brain } \\
\text { lesion (TH, GB Channels) } \\
\text { Step 5: GV20, GB20, GV16, Yintang }\end{array}$ & $\begin{array}{l}1 / \text { week } \\
1 / \text { week }\end{array}$ & - & $\begin{array}{l}1 / 1 \\
4 / 5\end{array}$ \\
\hline
\end{tabular}

3.5. Cough $(n=66)$. The coughing cases in this study consisted of 60 shelter and 6 hospital cases. On August and September 2007 in Republic of Korea, workers in two shelters noticed abrupt outbreaks of coughing dogs after rainy spell in summer, although the medications including antibiotics were done under regular shelter program. The most affected dogs were small pure or mixed breeds. Although the exact age of the shelter dogs was usually unknown, ages were estimated to be between 6 months and 7 years old, based on dentition and hair coat. The common clinical signs were cough and nasal discharge for 3-7 days. Under shelter's approval, treatment was performed using acupuncture without Western medicine, at the following acupoints: LU7, KI6, and LI4 (in Step 1), CV17 or LV13 (in Step 2), sensitive Back-Shu and Abdomen-Mu points to palpations (in Step 3), LU9 and LU5 (in Step 4), and CV22 (in Step 5). Two shelter veterinarians observed daily spontaneous coughing and nasal discharge in acupuncture-treated dogs. At 7 days after one acupuncture treatment, it was noted that 48 dogs showed no symptoms of cough and nasal discharge, and the other 12 had still cough or nasal discharge and thereafter were prescribed antibiotics. The above favorable effects were also observed in 6 hospital cases (1 Poodle, 1 Yorkshire terrier, 3 Shih Tzus, and 1 mixedbreed) within 2-7 days at onset duration of coughing. They received acupuncture treatments two times a week at the same points as those of shelter dogs. Decrease of coughing frequencies was observed on 3 days after first acupuncture and they all did not show any coughing on the next visits (on 7 days after acupuncture treatment).

3.6. Epilepsy $(n=6)$. The 6 cases ( 2 Pugs, 1 Shih Tzu, 1 Maltese, 1 miniature Poodle, and 1 mixed breed) showed recurrent seizure at a frequency of $2-4 /$ month under control of antiepileptic drugs. They were diagnosed as presumed idiopathic epilepsy based on physical and neurological examinations and hematological and serum biochemical analyses. One Maltese dog of 3 months at onset of epileptic seizure received only acupuncture treatment once a week without medication, since his owner was reluctant to give antiepileptic drugs. The other 5 dogs over 1 year at onset of epileptic seizure received regular acupuncture treatment once a month with anticonvulsants. Acupoints were chosen, as shown in (Table 2). Since acupuncture treatment, 5 of the 6 dogs $(5 / 6,83 \%)$ had no epileptic episode during followup of 12 months. However, a 5-year-old male Pug, presented with frequent generalized and symmetrical epilepsy during 1.5 years, did not show any changes in frequencies of epilepsy after acupuncture treatment.

\section{Discussion}

4.1. The Present Acupuncture Flow Chart Follows Basic OM Principles for Point Combinations. Although various methods can be used to select effective acupoints in OM, good 
prescriptions must satisfy at least basic OM principles. These include (1) bilateral acupuncture, (2) combination of forelimb and hindlimb points, (3) ventral and dorsal points, and (4) local and distal points [7]. The present chart follows all of the above principles. First, each step is bilateral acupuncture. Second, Step 1 includes a combination of points on forelimb and hindlimb. Third, Step 3 is one technique of combining $M u$-ventral and Shu-dorsal points. For example, in vomiting cases, ventral point CV12 and dorsal point BL21 were selected in Step 3. Lastly, a combination of local and distal points is included in Steps 4 and 5. Thus, the present flow chart is well matched with OM principles for point combination.

4.2. The Proposed Chart Lists 164 Important Acupoints and Is Designed to Select Acupoints in a Total of 5 Steps. There are approximately 360 acupoints on the 14 main channels of the body. Not all these acupoints are used commonly in veterinary and human clinics. Most acupuncturists pay great attention to the application of special acting acupoints which have special therapeutic effects. These clinically important acupoints are categorized according to their own special therapeutic properties as follows: 8 Extraordinary Channel points, 6 Command points, 8 Influential points, Back-Shu and Abdomen-Mu points, Yuan (source) points and Luo (connection) points, and 5 Shu (transporting) points, including $\mathrm{He}$ (sea) points, $\mathrm{Xi}$ (cleft) points, and empirical points, and local points. In the present chart, the above points were organized in order of the functional ranges of acupoints (from points with broad function to points acting locally) as follows (Figure 1).

Step 1: 8 Extraordinary Channel points (8 points) +6 Command points (6 points),

Step 2: 8 Influential points (8 points),

Step 3: Back-Shu points (12 points) + Abdomen-Mu points (12 points),

Step 4: 5 Shu (transporting) points on 12 Channels (55 points) $+X i$ (cleft) points (12 points) + Yuan (source) points (12 points) + Luo (connection) points (12 points),

Step 5: empirical or local points (27 points).

In Step 1, Extraordinary Channel points were combined with Command points because both acupoints groups have the widest range of actions among the acupoints and have similar indications in respects of the body areas, when divided the body into exterior (lateral or back aspects) and interior (heart/gastrointestinal or respiratory/urogenital areas) or upper (face and neck) and lower parts (back). In oriental medicine, 8 Extraordinary Channels and their key points have been considered to play most important roles in balancing body $Q i$. The medical term " 8 Extraordinary Channels points" dates back to 1230 s and the detailed indications and methods forming 4 pairs of acupoints (i.e., PC6-SP4) were first described in the Yizong Jinjian (Golden Mirror of Medicine) written by $W u$ Qian in 1742 [8]. Based on that book, the Extraordinary Channel points are always used in a pair for disorders of the following body areas: (1) SI3BL62-back, spine, neck, head, eye, and brain; (2) TH5-GB41side of body, lateral sides of the lumbar area, lateral aspect of leg, sides of body, shoulders, hip, eyes, ears, and neck; (3) PC6-SP4-heart, thorax, gastrointestinal disorders, and reproductive, and (4) LU7-KI6-respiratory, lower abdominal, and urogenital disorders $[9,10]$.

To simplify the above theory more, we divided the body into 4 areas of exterior-back aspects, exterior-lateral aspects, interior-heart/gastrointestinal and interior-respiratory/uro \genital systems, and assigned paired acupoints to each area (Figures 1 and 2(a)). In our clinical cases (Table 2), a pair of SI3-BL62 was applied to back disorders (IVDD) and bilateral epilepsy which was considered to be caused by posterior (or whole) brain lesions. A pair of TH5-GB41 was used for 1 case with unilateral epilepsy which was presumed to be due to lateral brain lesions, and LU7-KI6 was chosen for 66 coughing cases. And PC6-SP4 points were used for vomiting $(n=11)$, diarrhea $(n=2)$ and abdominal pain $(n=5)$. Previous experimental and clinical studies have successfully applied 8 Extraordinary Channel points. In one clinical study showing successful outcomes of acupuncture in IVDD dogs, paired acupoints of SI3-BL62 were used in combination with other acupoints [11]. TH5-GB41 has been used effectively for unilateral or focal headaches in human $[12,13]$. LU7 and/or KI6 in combination with other acupoints has been used successfully for respiratory clinical trials [14-16]. Acupoint PC6 is extensively for treatment and prevention of vomiting [17-23] and the enhancement of cardiopulmonary functions [4, 24-26]. Wang et al. reported that acupoints at PC6 and SP4 enhance cardiac and gastrointestinal functional activities after acute myocardial ischemia through the mediation of nitric oxide (NO) [27].

In Step 1, theoretically, to enhance the effects of Extraordinary Channel points, 6 Command points therapy were added. In detail, the 6 Command points are 6 individual points which have been used to control diseases in 6 major body parts, abdomen (ST36), lumbar region (BL40), neck (LU7), heart (LI4), chest (PC6), and urogenital organ (SP6) [28] (Figure 2(b)). Six Command points originated from 4 Command points (ST36, LI4, LU7 and BL40), described in Chinese classic Qiankunshengyi (Meanings of Life between Heaven and Earth; 1402) and formed by adding two points (PC6 and SP6) later [29]. In the present study, one of 6 Command points was chosen additionally in cases of IVDD (BL40) and diarrhea/abdominal pain (ST36). Previous experimental and clinical studies support the effect of the 6 Command points which described in Step 1 of Figure 1. BL40 commonly in previous clinical trials of dogs with IVDD $[11,30]$. ST36 is a acupoint, well known to be most effective for gastrointestinal disorders such as abdominal pain, diarrhea, and irritable bowel diseases [3, 31-38]. Acupuncture at SP6, a common point for urinary disorders $[39,40]$, has shown to decrease symptoms of urinary incontinence by stress in rats [41], diurnal symptoms associated with idiopathic bladder instability [42], and symptoms of frequency, urgency, and dysuria in female cases [43]. Although we could not conclude that the application of these combined points in Step 1 led to the present favorable outcomes, at least this flow chart allows 
an easy and fast approach to apply clinically the complicated OM theory concerning 8 Extraordinary Channel and 6 Command points.

In Step 2, one or two of 8 Influential points were selected according to the body components affected by diseases. Eight Influential points are based on a Chinese classic Nan Jing (The Classic of Difficulties) written around the 2nd century AD. It classifies the body as having 8 components: Zang, $F u, Q i, X u e$ (Blood), Ji (Muscle), Mai (Vessels), Gu (Bone), and Sui (Marrow). Each of these has a most influential point that exerts a profound effect on the function of each component [44]. Figure 3 and Step 2 in Figure 1 show 8 body components in OM, interpretation in Western medicine, and their corresponding Influential points. For example, CV12 can be used for disorders of stomach, small intestine and large intestine ( $F u$ organs in $\mathrm{OM})$, such as epigastric distention, abdominal pain, constipation, or diarrhea, and GB34 can be selected for muscle-related disorders (muscle in OM) such as muscle spasm, painful tendons, and hemiplegia. In our present studies, we chose GB34 for IVDD cases, LV13/CV12 for vomiting, diarrhea, and abdominal pain, and CV17/LV13 for cough and GB39 for epilepsy, respectively. Previous studies have included acupoint(s) of GB34 in IVDD [11, 30], LV13/CV12 in gastrointestinal disorders [34, 45, 46], CV17 in respiratory disorders [47], and GB39 in brain disorders, respectively [48].

In Step 3, Back-Shu and Abdomen-Mu points showing sensitivity to surgeon's palpation were selected for acupuncture. Figure 4 and Step 3 in Figure 1 show the Back-Shu and Abdomen- $M u$ points and the associated internal organs. Oriental medicine describes that Back-Shu and Abdomen$M u$ points are connected directly to the internal organs and these points often become tender, tight, or distended when the associated organs are diseased or imbalanced, and so they are used as diagnostic and treatment points $[5,49]$. In support, a retrospective study of 175 dogs and cats with Back$S h u$ or Abdomen- $M u$ point sensitivity and their blood chemistry showed that there is at least a single correlation of the point sensitivity with a concurrent rise in the internal organsassociated chemistry values [49]. Back-Shu and Abdomen$M u$ points are known to be related segmentally to the internal organs [50,51]. Visceral pain is referred to segmental somatic areas. In the referred area, tender points, characterized by well-defined and localized spots and an increased sensitivity to mechanical stimuli are often found [52]. The stimulation of these tender points can in turn alleviate the visceral pain and inflammation [53-55]. In our present cases, most sensitive points were found at Back-Shu and Abdomen-Mu points around the affected internal organs and frequently some other points. In the cases with vomiting $(n=11)$ or abdominal pain $(n=5)$, sensitive points were found at BL21 (stomach Shu point) and/or CV12 (stomach $M u$ point) and frequently several other points such as BL17 (diaphragm point), BL18 (liver point), BL 19 (gall bladder point), BL 20 (spleen point), BL22, and BL23 (kidney point). Two diarrhea cases were sensitive at BL23/BL25 or BL21/BL24/BL25. In the cough cases, one or two sensitive points at Back-Shu points such as BL12 (wind point), BL13 (lung point), BL14 (pericardium point), or BL15 (heart point) were found. These above points were stimulated by acupuncture.

In Step 4, one or two of 12 regular Channels were chosen according to main symptoms or diagnosis from Step 3, and then two Main points (Yuan and He points) were selected on the chosen Channels (Figure 1). Regular Channels are Qi pathways to connect the external body with internal organs (Yin Channels) or head (Yang Channels) [5]. Oriental medicine teaches that diseases occur when the $Q i$ flow is disrupted in one or more Channels, which can be relieved by stimulating acupoints on the affected Channels. To differentiate the Channels which were likely to be affected by disorders, we used the "3 Yang-3 Yin theory" which has been the most important fundament for diagnosis and treatment in acupuncture medicine [56, 57] (Figure 5(a)). In ancient anatomical terms, Yang and Yin represent exterior and interior of body, respectively. In respect of treatment, Yang and Yin also represent external (exterior) and internal (interior) disorders, respectively. The Yang (exterior of body) is divided into 3 sub-Yangs, namely, Yangming (front), Shaoyang (lateral) and Taiyang (back or dorsal), and each of them is used for its corresponding external disorders (Figures 5(a) and 5(b) and Step 4 in Figure 1). In our present study, based on "3 Yang-3 Yin theory," IVDD or epilepsy was diagnosed as exterior-back (Taiyang) disorder and thus Taiyang Channels (SI and BL) were chosen. Then, two Main points Yuan (source) and $\mathrm{He}$ (sea) points on SI and BL Channels were selected for acupuncture. Shaoyang Channels (TH and GB) were chosen for epilepsy with clinically presumed unilateral brain lesion (Table 2). On the other hand, the Yin (interior of body) is divided into 3 sub-Yins, namely, Taiyin (front organs; lung, spleen), Jueyin (middle organs; pericardium, liver) and Shaoyin (dorsal organs; heart, kidney), and each of them is used for treatment of its specific internal organs (Figures 5(a) and 5(c)). Empirically, stomach (ST) Channel of Yang Channels has been used to treat internal organs (stomach and intestine), as well as exterior-front disorder. In our present study, cough was diagnosed as interior-front organ-Lung (Taiyin LU) disorder, and thus Taiyin LU Channel and its Main points (Yuan and He points) were selected for treatment. Case with vomiting, diarrhea, or abdominal pain was diagnosed as interior-front organ-spleen (Taiyin SP) disorder or stomach (ST) disorder, according to sensitivity at BL20 (spleen Shu point) or BL21 (stomach Shu point) and two Main points were selected on SP (spleen) or ST (stomach) Channels (Table 2). Although the above Channel theory is most important in acupuncture medicine, it is true that the theory is too difficult for general clinicians to comprehend. Therefore, we highly simplified clinical indications of each Channel and summarized it in Step 4 (Figure 1).

In Step 5, local, empirical, or Ashi (local sensitive) points for each case were added. Acupuncture needling is known to produce local anti-inflammatory, analgesic, and antipyretic effects by promoting vasodilation and blood flow locally and releasing neuromodulators [58]. In the present cases, we chose GV1 in diarrhea, CV22 in cough, and GV20/GB20/ GV16/Yintang in epilepsy, respectively. GV1 is a single acupoint in the depression ventral to the base of the tail and dorsal to the anus. It is one of the most effective acupoints to 
treat diarrhea in humans and animals [59-61]. Our previous studies demonstrated that acupuncture at GV1 depressed proximal colonic motility by decreasing the total duration and frequency of contractile states in conscious dogs and also had anti-inflammatory and analgesic effects in colitis rats, via endogenous opioid pathways $[54,55,62]$. CV22 has been included to treat respiratory disorders $[63,64]$. GV20, GB20, GV16, or Yintang points have been used commonly for brain disorders such as headache [65]. Previous reports has shown that acupuncture at Yintang and/or GV20 can cause sedative effects and change bioelectrical brain activity [66]. Although we could not determine which acupoint in flow chart was most effective for the treatment of each case, each step in this flow chart provides a theoretical rationale for selection of optimal acupoints in each case, based on OM theory.

\subsection{The Proposed Chart Can Help Clinicians Prescribe Acu-} points Effectively for Various Diseases. In 1997, the $\mathrm{NIH}$ released a consensus statement concluding that acupuncture is effective or at least useful for the treatment of 13 conditions including low back pain, nausea and vomiting, asthma, stroke rehabilitation, headache, addiction, dental pain, menstrual cramps, tennis elbow, fibromyalgia, myofascial pain, osteoarthritis, and carpal tunnel syndrome [67]. Multiple studies have documented that acupuncture is useful in the case with low back pain or IVDD when a definitive diagnosis is made or when surgical intervention is not an option due to patient concerns such as geriatric and other underlying diseases, and acupuncture results are favorable and comparable to those of surgical treatments [30, 68-75]. In veterinary medicine, the success rates and recovery periods by acupuncture in IVDD dogs seem to vary according to the severity of disease. In clinical reports of Janssens LA [76], 90\% of dogs with grade I recovered after 2-3 treatment over 1-2 week period, $90 \%$ of dogs with grade II recovered after 3-4 treatment over a 3-week period, and $80 \%$ dogs with grade III recovered after 5-6 treatments over a 6-week period. And dogs with grade IV showed poor response to acupuncture (success rate $<25 \%$ ). Similarly, in our present study, $100 \%$ in dogs of grades I to III recovered over periods from 5 days to 6 weeks after 1-12 treatments, while only $1 / 4$ dogs in grade IV recovered over 7 weeks after 15 treatments. It seemed that as the severity of IVDD increased, the recovery period and number of treatments also increased. Acupuncture treatment of idiopathic epilepsy has been documented well in the veterinary and human literature $[5,57,68]$. It was reported that acupuncture reduces seizure frequency and dosage requirements of antiepileptic drugs in epileptic dogs [77-80]. In our present study, 5/6 dogs with epileptic episode at a frequency of $2-4 /$ month showed no seizures under combination therapy of acupuncture and anticonvulsants. It indicates that this flow chart may be useful for neurological cases with IVDD or epilepsy.

Acupuncture is used extensively for gastrointestinal disorders, such as vomiting, diarrhea, and abdominal pain. It decreases the severity of nausea and emesis from a variety of causes in humans and dogs [18, 20, 21,67]. In the present study, all dogs with vomiting $(n=11)$, diarrhea $(n=2)$, and abdominal pain $(n=5)$ recovered after $1-6$ treatments over 119 days. Most acute/subacute cases ( $<7$ days at duration) fully recovered within 1 day after one acupuncture treatment, without Western medicine. Interestingly 3/6 acute vomiting cases began to eat foods within $1 \mathrm{hr}$ after withdrawal of acupuncture needles and 4/5 abdominal pain cases showed complete relief of abdominal pain within $30 \mathrm{~min}$ after withdrawal of acupuncture needles, with no recurrence. Similarly, in one human clinical study of 190 cases with intestinal colic pain from bacillary dysentery, simple acute appendicitis, simple acute intestinal obstruction, adhesive intestinal obstruction, or intestinal ascariasis, acupuncture at bilateral ST36 results in complete pain relief within $30 \mathrm{~min}$ in $85 \%$ of cases, decreased colic pain in $11 \%$, and no response in $4 \%$ [81]. To our knowledge, although it has limitations to explain these phenomena scientifically, our results show that this flow chart can help the clinicians prescribe acupoints effectively for cases with various gastrointestinal symptoms. Acupuncture may have beneficial effects on the treatment of respiratory diseases including bronchitis and asthma in dogs and cats [82]. Although this was an uncontrolled clinical study, and not a randomised controlled clinical trial, our results that acupuncture improved 54/66 coughing cases showed that acupuncture methods using this flow chart can be used to help the treatment of respiratory cases.

\section{Conclusion}

This acupoints prescription chart is based on oriental medical (OM) theory and includes information concerning OM diagnosis, function of the Channels, Zang-Fu theory, clinically important acupoints, and combination methods of their acupoints. It has been modified through clinical trials since first edition in 2003 and used widely and successfully to various clinical cases such as neurological, respiratory, and gastrointestinal disorders in Korean veterinary clinics. We believe that this chart helps beginners or clinicians to select effective acupuncture points easily and quickly. However, more powerful methods of well-designed randomized and controlled trials are needed to confirm the efficacy of this flow chart on various diseases.

\section{Acknowledgments}

This work was supported by the National Research Foundation of Korea (NRF) grant funded by the Korea Government (MEST) (no. 20120009400). The authors thank Phil Rogers MRCVS, Dublin, for editing the material in this paper. Hee Young Kim also appreciates and acknowledges the support for the basic course on veterinary acupuncture (2007-2008, San Antonio, USA) provided by the International Veterinary Acupuncture Society (IVAS) via the Grady Young Memorial Scholarship program.

\section{References}

[1] D. H. Jaggar and N. G. Robinson, "History of veterinary acupuncture," in Veterinary Acupuncture: Ancient Art to Modern Medicine, A. M. Schoen, Ed., pp. 3-17, Mosby, St. Louis, Mo, USA, 2002. 
[2] A. Shiotani, M. Tatewaki, E. Hoshino, and T. Takahashi, "Effects of electroacupuncture on gastric myoelectrical activity in healthy humans," Neurogastroenterology and Motility, vol. 16, no. 3, pp. 293-298, 2004.

[3] S. Xu, Z. Liu, and M. Xu, "Treatment of cancerous abdominal pain by acupuncture on zusanli (ST 36) - a report of 92 cases," Journal of Traditional Chinese Medicine, vol. 15, no. 3, pp. 189191, 1995.

[4] M. T. Tsou, C. H. Huang, and J. H. Chiu, "Electroacupuncture on PC6 (Neiguan) attenuates ischemia/reperfusion injury in rat hearts," The American Journal of Chinese Medicine, vol. 32, no. 6, pp. 951-965, 2004.

[5] H. Kim, I. Shim, D. Hahm et al., Canine Acupuncture, Korvet, Seoul, Republic of Korea, 2004.

[6] J. R. Coates, "Intervertebral disk disease," Veterinary Clinics of North America-Small Animal Practice, vol. 30, no. 1, pp. 77$110,2000$.

[7] M. H. Glinski, "Point selection," in Veterinary Acupuncture: Ancient Art to Modern Medicine, A. M. Schoen, Ed., pp. 113-124, Mosby, St. Louis, Mo, USA, 2001.

[8] G. S. Lee and J. H. Yoon, "Study about eight extraordinary vessels and eight extraordinary vessels points," Journal of Korean Academy of Medical Gi-Gong, vol. 2, no. 1, pp. 103-118, 1998.

[9] G. Maciocia, The Foundations of Chinese Medicine: A Comprehensive Text for Acupuncturists and Herbalists, Churchill Livingstone, 1989.

[10] J. Y. Jang, S. Y. Park, J. A. Hong et al., "A literary study on combination of Yeolgyeol (LU7) and Johae (KI6) of eight confluent acupoints," The Korean Journal of Meridian and Acupoint, vol. 23, pp. 27-47, 2006.

[11] A. M. Hayashi, J. M. Matera, and A. C. B. D. C. F. Pinto, "Evaluation of electroacupuncture treatment for thoracolumbar intervertebral disk disease in dogs," Journal of the American Veterinary Medical Association, vol. 231, no. 6, pp. 913-918, 2007.

[12] A. Kuruvilla, "Acupuncture outcome of patients with migraine," Medical Acupuncture, vol. 19, no. 3, pp. 137-140, 2007.

[13] M. Karst, M. Reinhard, P. Thum, B. Wiese, J. Rollnik, and M. Fink, "Needle acupuncture in tension-type headache: a randomized, placebo-controlled study," Cephalalgia, vol. 21, no. 6, pp. 637-642, 2001.

[14] E. Peuker, "Case report of tension pneumothorax related to acupuncture," Acupuncture in Medicine, vol. 22, no. 1, pp. 4043, 2004.

[15] W. Zwölfer, W. Keznickl-Hillebrand, A. Spacek, M. Cartellieri, and G. Grubhofer, "Beneficial effect of acupuncture on adult patients with asthma bronchiale," The American Journal of Chinese Medicine, vol. 21, no. 2, pp. 113-117, 1993.

[16] A. O. Freire, G. C. M. Sugai, F. S. Chrispin et al., "Treatment of moderate obstructive sleep apnea syndrome with acupuncture: a randomised, placebo-controlled pilot trial," Sleep Medicine, vol. 8, no. 1, pp. 43-50, 2007.

[17] E. Nystrom, G. Ridderstrom, and A. S. Leffler, "Manual acupuncture as an adjunctive treatment of nausea in patients with cancer in palliative care-a prospective, observational pilot study," Acupuncture in Medicine, vol. 26, no. 1, pp. 27-32, 2008.

[18] G. Gardani, R. Cerrone, C. Biella et al., "A progress study of 100 cancer patients treated by acupressure for chemotherapyinduced vomiting after failure with the pharmacological approach," Minerva Medica, vol. 98, no. 6, pp. 665-668, 2007.

[19] G. Gardani, R. Cerrone, C. Biella et al., "Effect of acupressure on nausea and vomiting induced by chemotherapy in cancer patients," Minerva Medica, vol. 97, no. 5, pp. 391-394, 2006.
[20] M. Tatewaki, C. Strickland, H. Fukuda et al., "Effects of acupuncture on vasopressin-induced emesis in conscious dogs," The American Journal of Physiology, vol. 288, no. 2, pp. R401R408, 2005.

[21] H. E. Dent, N. G. Dewhurst, S. Y. Mills, and M. Willoughby, "Continuous PC6 wristband acupressure for relief of nausea and vomiting associated with acute myocardial infarction: a partially randomised, placebo-controlled trial," Complementary Therapies in Medicine, vol. 11, no. 2, pp. 72-77, 2003.

[22] C. P. O. Carlsson, P. Axemo, A. Bodin et al., "Manual acupuncture reduces hyperemesis gravidarum: a placebo-controlled, randomized, single-blind, crossover study," Journal of Pain and Symptom Management, vol. 20, no. 4, pp. 273-279, 2000.

[23] M. Al-Sadi, B. Newman, and S. A. Julious, "Acupuncture in the prevention of postoperative nausea and vomiting," Anaesthesia, vol. 52, no. 7, pp. 658-661, 1997.

[24] J. G. Lin, S. J. Ho, and J. C. Lin, "Effect of acupuncture on cardiopulmonary function," Chinese Medical Journal, vol. 109, no. 6, pp. 482-485, 1996.

[25] J. Gao, W. Fu, Z. Jin, and X. Yu, "A preliminary study on the cardioprotection of acupuncture pretreatment in rats with ischemia and reperfusion: involvement of cardiac $\beta$-adrenoceptors," Journal of Physiological Sciences, vol. 56, no. 4, pp. 275-279, 2006.

[26] J. Gao, W. Fu, Z. Jin, and X. Yu, "Acupuncture pretreatment protects heart from injury in rats with myocardial ischemia and reperfusion via inhibition of the $\beta 1$-adrenoceptor signaling pathway," Life Sciences, vol. 80, no. 16, pp. 1484-1489, 2007.

[27] S. B. Wang, S. P. Chen, Y. H. Gao, M. F. Luo, and J. L. Liu, "Effects of electroacupuncture on cardiac and gastric activities in acute myocardial ischemia rats," World Journal of Gastroenterology, vol. 14, no. 42, pp. 6496-6502, 2008.

[28] H. C. Jeong and I. C. Sohn, "A literary study of four command points," The Journal of Korean Acupuncture and Moxibustion Society, vol. 13, no. 1, pp. 292-306, 1996.

[29] Y. An, All Guide of Acupuncture, 2002.

[30] S. M. Jeong and S. W. Park, "Application of traditional acupuncture on canine intervertebral disc disease," Journal of Veterinary Clinics, vol. 21, no. 1, pp. 49-52, 2004.

[31] K. M. Cui, W. M. Li, X. Gao, K. Chung, J. M. Chung, and G. C. $\mathrm{Wu}$, "Electro-acupuncture relieves chronic visceral hyperalgesia in rats," Neuroscience Letters, vol. 376, no. 1, pp. 20-23, 2005.

[32] J. Xing, B. Larive, N. Mekhail, and E. Soffer, “Transcutaneous electrical acustimulation can reduce visceral perception in patients with the irritable bowel syndrome: a pilot study," Alternative Therapies in Health and Medicine, vol. 10, no. 1, pp. 38-42, 2004.

[33] M. T. Cabýoglu, N. Ergene, and U. Tan, "The mechanism of acupuncture and clinical applications," International Journal of Neuroscience, vol. 116, no. 2, pp. 115-125, 2006.

[34] H. Qin-Feng and Q. Li-Zhen, "Treatment of chronic gastritis by acupuncture: a review," Journal of Acupuncture and Tuina Science, vol. 3, no. 2, pp. 7-8, 2005.

[35] M. Iwa, Y. Nakade, T. N. Pappas, and T. Takahashi, "Electroacupuncture improves restraint stress-induced delay of gastric emptying via central glutaminergic pathways in conscious rats," Neuroscience Letters, vol. 399, no. 1-2, pp. 6-10, 2006.

[36] M. Iwa, M. Matsushima, Y. Nakade, T. N. Pappas, M. Fujimiya, and T. Takahashi, "Electroacupuncture at ST-36 accelerates colonic motility and transit in freely moving conscious rats," The American Journal of Physiology, vol. 290, no. 2, pp. G285-G292, 2006. 
[37] L. Tian, Y. X. Huang, M. Tian, W. Gao, and Q. Chang, "Downregulation of electroacupuncture at ST36 on TNF- $\alpha$ in rats with ulcerative colitis," World Journal of Gastroenterology, vol. 9, no. 5, pp. 1028-1033, 2003.

[38] D. Luo, S. Liu, X. Xie, and X. Hou, "Electroacupuncture at acupoint ST-36 promotes contractility of distal colon via a cholinergic pathway in conscious rats," Digestive Diseases and Sciences, vol. 53, no. 3, pp. 689-693, 2008.

[39] W. M. Yi, J. J. Li, X. M. Lu, L. L. Jin, A. Z. Pan, and Y. Q. Zou, "Effects of electroacupuncture on urinary bladder function after radical hysterectomy," Zhongguo Zhen Jiu, vol. 28, no. 9, pp. 653655, 2008.

[40] S. O. Aschkenazi and P. K. Sand, Alternative Therapies for Urinary Urgency Incontinence: Acupuncture and Herbology, Springer, London, UK, 2009.

[41] I. M. Chung, Y. S. Kim, Y. H. Sung et al., "Effects of acupuncture on abdominal leak point pressure and c-Fos expression in the brain of rats with stress urinary incontinence," Neuroscience Letters, vol. 439, no. 1, pp. 18-23, 2008.

[42] T. Philp, P. J. R. Shah, and P. H. L. Worth, "Acupuncture in the treatment of bladder instability," The British Journal of Urology, vol. 61, no. 6, pp. 490-493, 1988.

[43] P. L. Chang, C. J. Wu, and M. H. Huang, "Long-term outcome of acupuncture in women with frequency, urgency and dysuria," The American Journal of Chinese Medicine, vol. 21, no. 3-4, pp. 231-236, 1993.

[44] B. H. Lee and J. Y. Moon, "The study of meaing and formation system of Pal Hyue Hyul (eight influential points) through Hwangjaenaekyong," The Journal of Korean Acupuncture and Moxibustion Society, vol. 22, no. 6, pp. 101-109, 2005.

[45] T. Takahashi, "Acupuncture for functional gastrointestinal disorders," Journal of Gastroenterology, vol. 41, no. 5, pp. 408-417, 2006.

[46] Y. S. Kim, K. W. Kim, J. Y. Kim et al., "The anti-emetic effect of needling acupuncture, aquapuncture and moxibustion at BL21 and CV-12 in xylazine induced vomiting of dogs," Journal of Veterinary Clinics, vol. 22, no. 3, pp. 202-205, 2005.

[47] S. Y. Zhang, "Treatment of 200 cases of asthma by fat-cut therapy," Journal of Acupuncture and Tuina Science, vol. 3, no. 3, pp. 24-25, 2005.

[48] C. H. Lee, T. H. Kim, S. Y. Lee et al., "Neuroanatomical studies on acupoints(SJ5, Pe6, SP6 and GB39) projecting to the brain area related to dimentia using neural tracer, pseudorabies virus in mouse," The Journal of Korean Acupuncture and Moxibustion Society, vol. 20, no. 6, pp. 168-181, 2003.

[49] C. Schwarz, "Traditional Chinese medical diagnosis in small animals," in Veterinary Acupuncture: Ancient Art to Modern Medicine, A. M. Schoen, Ed., pp. 149-160, Mosby, St. Louis, Mo, USA, 2001.

[50] D. F. Mayor, "The Chinese back shu and front mu points and their segmental innervation," Deutsche Zeitschrift fur Akupunktur, vol. 51, no. 2, pp. 26-36, 2008.

[51] W. Cai, "Acupuncture and the nervous system," The American Journal of Chinese Medicine, vol. 20, no. 3-4, pp. 331-337, 1992.

[52] M. A. Giamberardino, "Recent and forgotten aspects of visceral pain," European Journal of Pain, vol. 3, no. 2, pp. 77-92, 1999.

[53] B. Coffin, F. Azpiroz, and J. R. Malagelada, "Somatic stimulation reduces perception of gut distention in humans," Gastroenterology, vol. 107, no. 6, pp. 1636-1642, 1994.

[54] H. Y. Kim, D. H. Hahm, K. H. Pyun et al., "Effects of acupuncture at GV01 on experimentally induced colitis in rats: possible involvement of the opioid system," Japanese Journal of Physiology, vol. 55, no. 3, pp. 205-210, 2005.

[55] H. Y. Kim, D. H. Hahm, B. Y. Sohn et al., "Skin on GV01 acupoint in colonic inflammatory states: tenderness and neurogenic inflammation," Journal of Physiological Sciences, vol. 56, no. 4, pp. 317-320, 2006.

[56] C. S. Yin, M. K. Kang, S. H. Lee et al., "A study of three pairs of Yin and Yang acupoints in Dunhuang books on ancient medicine," The Journal of Korean Acupuncture and Moxibustion Society, vol. 19, no. 4, pp. 42-48, 2002.

[57] S. B. Kang, Acupuncture and Moxibustion Based on Ancient Books, Iljung, Seoul, Republic of Korea, 2000.

[58] J. E. Steiss, "The neurophysiologic basis of acupuncture," in Veterinary Acupuncture, A. Schoen, Ed., pp. 27-46, Mosby, St. Louis, Mo, USA, 2001.

[59] E. S. Park, S. Jo, J. K. Seong et al., "Effect of acupuncture in the treatment of young pigs with induced Escherichia coli diarrhea," Journal of Veterinary Science, vol. 4, no. 2, pp. 125-128, 2003.

[60] Y. C. Hwang and E. M. Jenkins, "Effect of acupuncture on young pigs with induced enteropathogenic Escherichia coli diarrhea," American Journal of Veterinary Research, vol. 49, no. 9, pp. 1641$1643,1988$.

[61] K. J. Jang, "A bibliographic study on the research of meridian point GV1," Journal of Korea Acupuncture and Moxibustion Society, vol. 13, pp. 384-391, 1996.

[62] H. Y. Kim, D. H. Hahm, Y. Chae et al., "Acupuncture at GV01 relieves somatic pain referred by colitis in rats," Journal of Physiological Sciences, vol. 57, no. 4, pp. 253-258, 2007.

[63] L. Z. Qi, Q. F. Huang, and L. Li-gong, "Treatment of asthma by acupuncture: a review," Journal of Acupuncture and Tuina Science, vol. 3, no. 3, pp. 3-5, 2005.

[64] H. Xie, Acupuncture for Internal Medicine, John Wiley \& Sons, New York, NY, USA, 2007.

[65] R. R. Coeytaux, W. Chen, C. E. Lindemuth, Y. Tan, and A. C. Reilly, "Variability in the diagnosis and point selection for persons with frequent headache by traditional Chinese medicine acupuncturists," Journal of Alternative and Complementary Medicine, vol. 12, no. 9, pp. 863-872, 2006.

[66] G. Litscher, "Ten years evidence-based high-tech acupuncture part 3: a short review of animal experiments," Evidence-Based Complementary and Alternative Medicine, vol. 7, no. 2, pp. 151155, 2010.

[67] S. S. Morey, "NIH issues consensus statement on acupuncture," The American Family Physician, vol. 57, no. 10, pp. 2545-2546, 1998.

[68] K. L. Kline, "Complementary and alternative medicine for neurologic disorders," Clinical Techniques in Small Animal Practice, vol. 17, no. 1, pp. 25-33, 2002.

[69] T. D. Scavelli and A. Schoen, "Problems and complications associated with the nonsurgical management of intervertebral disc disease," Problems in Veterinary Medicine, vol. 1, no. 3, pp. 402-414, 1989.

[70] L. A. Janssens, "Acupuncture for the treatment of thoracolumbar and cervical disc disease in the dog," Problems in Veterinary Medicine, vol. 4, no. 1, pp. 107-116, 1992.

[71] J. Still, "A clinical study of auriculotherapy in canine thoracolumbar disc disease," Journal of the South African Veterinary Association, vol. 61, no. 3, pp. 102-105, 1990.

[72] L. A. Janssens and P. A. Rogers, "Acupuncture versus surgery in canine thoracolumbar disc disease," Veterinary Record, vol. 124, no. 11 , article $283,1989$. 
[73] R. Buchli, "Successful acupuncture treatment of a cervical disc syndrome in a dog," Veterinary Medicine, Small Animal Clinician, vol. 70, no. 11, article 1302, 1975.

[74] A. M. Lubbe, "Auriculotherapy in canine thoracolumbar disc disease," Journal of the South African Veterinary Association, vol. 61, no. 4, article 187, 1990.

[75] A. M. Hayashi, J. M. Matera, T. S. da Silva, A. C. B. D. C. F. Pinto, and S. R. G. Cortopassi, "Electro-acupuncture and Chinese herbs for treatment of cervical intervertebral disk disease in a dog," Journal of Veterinary Science, vol. 8, no. 1, pp. 95-98, 2007.

[76] L. A. Janssens, "Acupuncture for thoracolumbar and cervical disk disease," in Veterinary Acupuncture: Ancient Art to Modern Medicine, A. M. Schoen, Ed., pp. 193-198, Mosby, St. Louis, Mo, USA, 2002.

[77] J. van Niekerk and N. Eckersley, "The use of acupuncture in canine epilepsy," Journal of the South African Veterinary Association, vol. 59, no. 1, article 5, 1988.

[78] A. M. Klide, G. C. Farnbach, and S. M. Gallagher, "Acupuncture therapy for the treatment of intractable, idiopathic epilepsy in five dogs," Acupuncture and Electro-Therapeutics Research, vol. 12, no. 1, pp. 71-74, 1987.

[79] T. E. Durkes, "Gold bead implants," Problems in Veterinary Medicine, vol. 4, no. 1, pp. 207-211, 1992.

[80] R. B. Panzer and C. L. Chrisman, "An auricular acupuncture treatment for idiopathic canine epilepsy: a preliminary report," The American Journal of Chinese Medicine, vol. 22, no. 1, pp. 1117, 1994.

[81] R. Jiang, "Analgesic effect of acupuncture on acute intestinal colic in 190 cases," Journal of Traditional Chinese Medicine, vol. 10, no. 1, pp. 20-21, 1990.

[82] C. Schwartz, "Chronic respiratory conditions and acupuncture therapy," Problems in Veterinary Medicine, vol. 4, no. 1, pp. 136$143,1992$. 


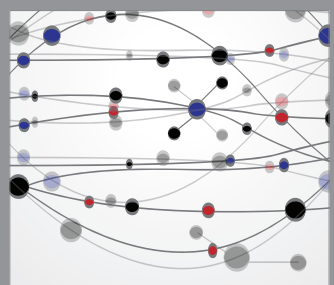

The Scientific World Journal
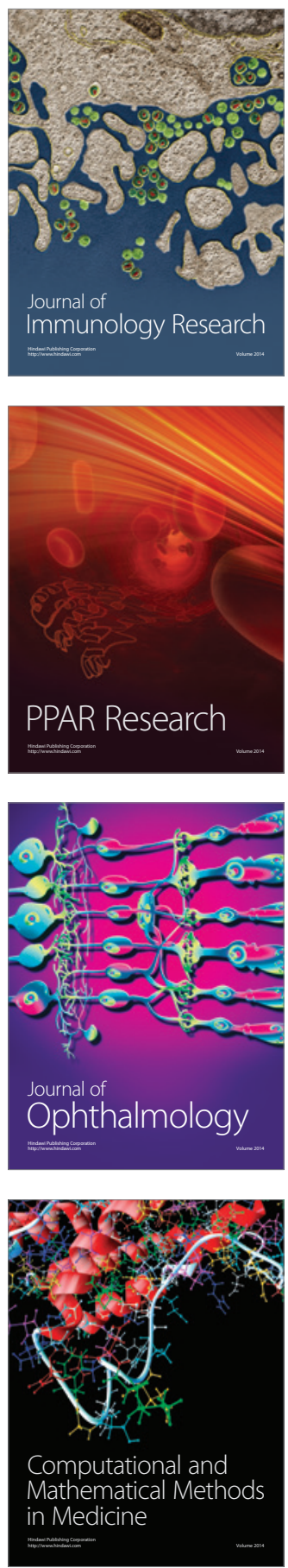

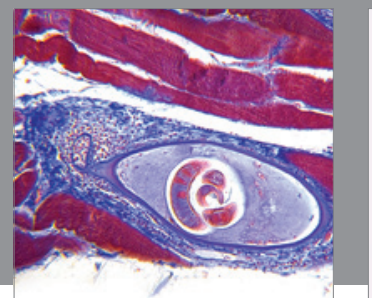

Gastroenterology

Research and Practice
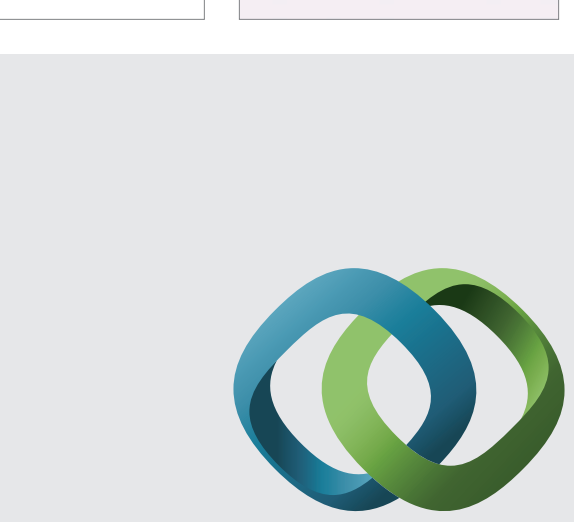

\section{Hindawi}

Submit your manuscripts at

http://www.hindawi.com
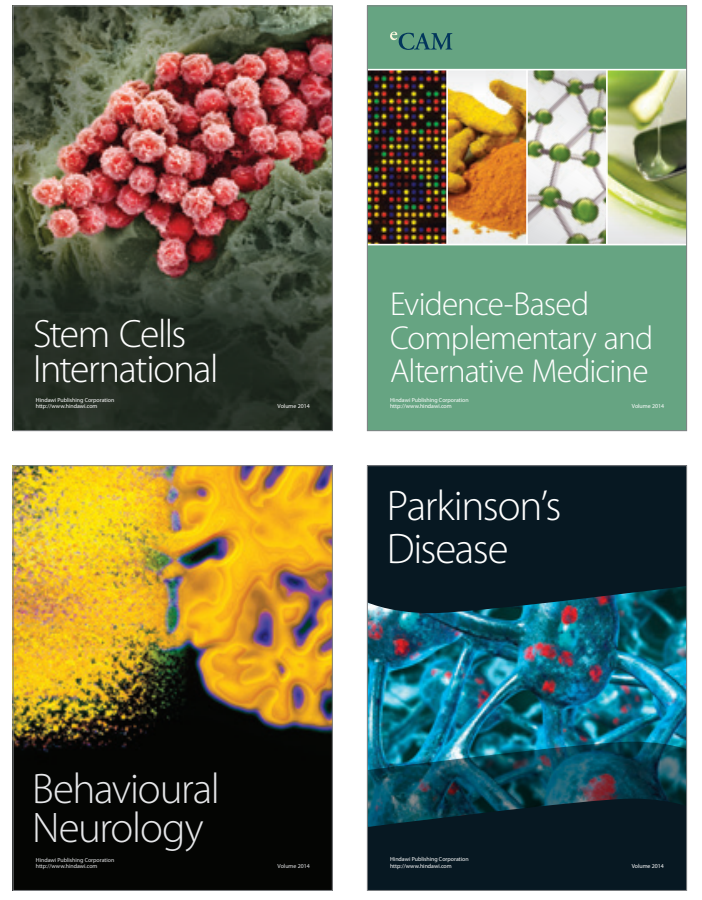
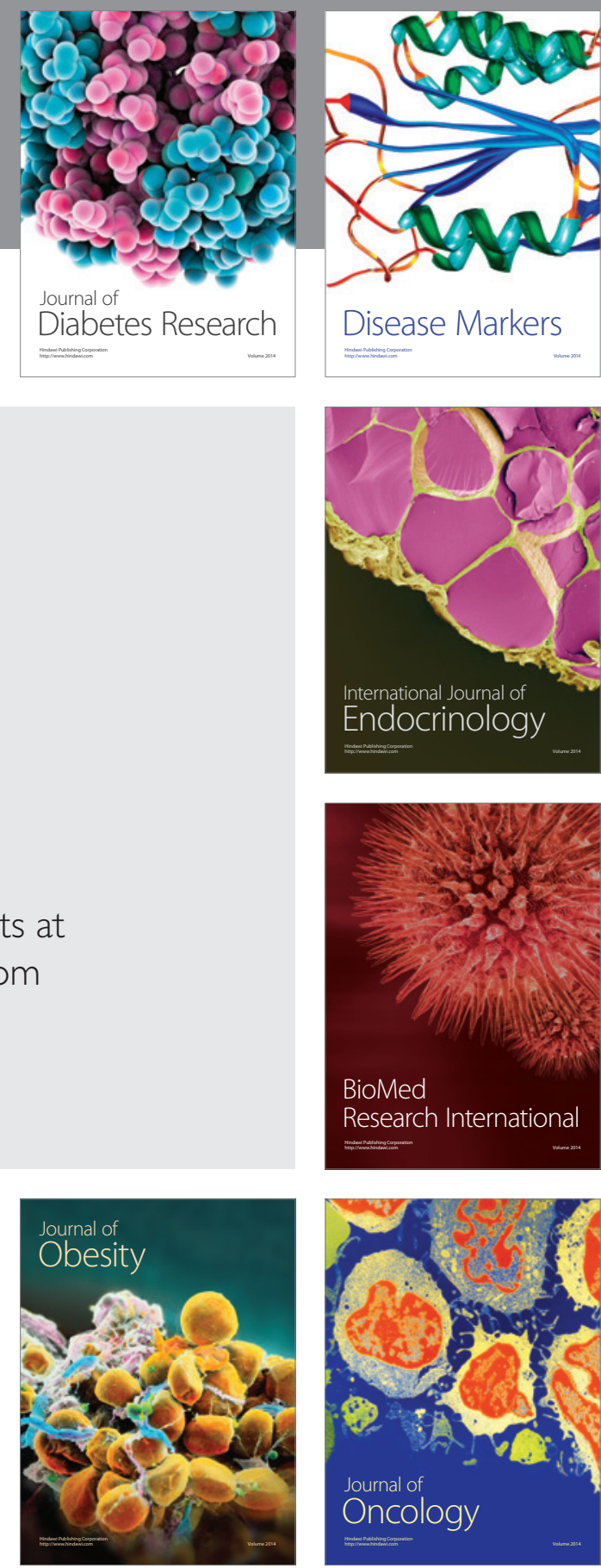

Disease Markers
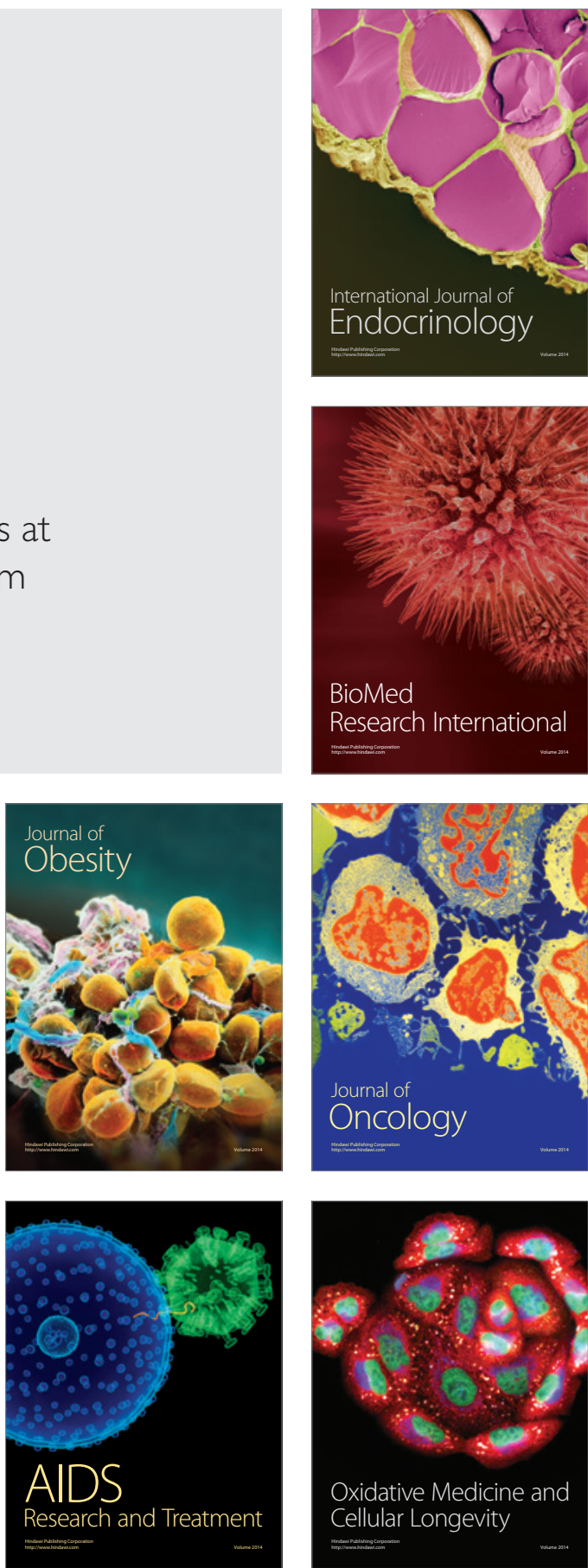